\title{
A CROSS-LAYER BASED SCALABLE CHANNEL SLOT RE-UTILIZATION TECHNIQUE FOR WIRELESS MESH NETWORK
}

\author{
Asha $\mathrm{C} \mathrm{N}^{1}$ and T G Basavaraju ${ }^{2}$ \\ ${ }^{1}$ VTU Research Scholar, Department of Electronics and communication \\ Engineering, \\ Acharya Institute of Technology, Bangalore, India \\ ashacn30@gmail.com \\ ${ }^{2}$ T G Basavaraju, Professor in CSE, Govt. SKSJTI, Bangalore, India \\ tg.braju@gmail.com
}

\begin{abstract}
Due to tremendous growth of the wireless based application services are increasing the demand for wireless communication techniques that use bandwidth more effectively. Channel slot reutilization in multi-radio wireless mesh networks is a very challenging problem. WMNs have been adopted as back haul to connect various networks such as Wi-Fi (802.11), WI-MAX (802.16e) etc. to the internet. The slot re-utilization technique proposed so far suffer due to high collision due to improper channel slot usage approximation error. To overcome this here the author propose the cross layer optimization technique by designing a device classification based channel slot re-utilization routing strategy which considers the channel slot and node information from various layers and use some of these parameters to approximate the risk involve in channel slot re-utilization in order to improve the QoS of the network. The simulation and analytical results show the effectiveness of our proposed approach in term of channel slot re-utilization efficiency and thus helps in reducing latency for data transmission and reduce channel slot collision.
\end{abstract}

\section{KEYWORDS}

Multi-radio WMN, Radio channel measurement, Scheduling, Routing, Medium access control $(M A C)$

\section{INTRODUCTION}

In current era wireless network gain much attention; research is ongoing day-by-day to improve more and more wireless technologies. Wireless Mesh Network (WMN) s is a part of this technologies growth. IEEE 802.15.5 MAC standard for mesh communication in wireless personal area network. WMN has a quality of dynamically self-organized and it can self-configure which make it more popular than other wireless network. Multi-hop transmission is one more

David C. Wyld et al. (Eds) : CSITY, SIGPRO, AIFZ, NWCOM, DTMN, GRAPHHOC - 2016

pp. 109-119, 2016. (C) CS \& IT-CSCP 2016

DOI : $10.5121 /$ csit.2016.60410 
characteristic of WMN. In a wireless Mesh network, there is fixed infrastructure over fixed wireless network which is used by wireless host. Every wireless host is connected with any of the mesh node; some mesh node may have direct association with internet. Channel quality in WMN fluctuated due to Doppler Effect, fading and interference [1]. MAC provides the actual benefit of mesh network. MAC- layer protocol by default chooses the minimum available transmission rate and it does not protect from error. Mesh network based on CSMA/CA MAC protocol which has single hop transmission characteristics, cannot provide the quality of service for the application which is streaming in real time like voice calling, video calling, etc. CSMA/CA has some limitation due to which we need a new MAC protocol which gives better throughput, capacity and reduce delay. To achieve better QoS it is needed to use the Time division multiple access (TDMA) based approach in MAC layer. Total channel (frequency channel or single band) is split into time-frame slot in TDMA scheduling and assignment of transmitting slot to the node is done. Power drop, collision, data overhead is prevented by every time slot [2]. TDMA with distributed approach consist of two different procedures. In first procedure it based on Bellman ford algorithm [3], each node find a nearest feasible link, which are taken from two-hop routing information updated by neighbour node. In second approach nearest feasible schedule is used to analyze the global feasible schedule and it inform the availability of a new schedule to the entire node.

\section{RELATED WORK}

Number of MAC-layer multicast mechanism was purposed for mesh network to overcome the inefficiency of the network. Hop-by-hop recovery on loss of packet is provided by researchers in various ways. Here [4] author gives the analysis of maximized output for a wireless mesh network over CSMA/CA in MAC layer protocol. Random access is not accounted in CSMA due to which collision overhead is increased. New development for optimal capacity analysis of network done in CSMA/CA with multi commodity flow (MCF), author analyze throughput based on upper and lower bound of the network capacity over CSMA/CA. the drawback of CSMA/CA is that it is not suitable for real time data transfer like video calling. In [5] if physical rate is increases efficiency of the MAC layer is decreases. More efficient MAC layer protocol in terms of scalability still has issues. Proposed scheme by author is MAC protocol based on dual channel token called (DT-MAC). This protocol is suitable for large number of user in terms of scalability and efficiency. Token management is extra overhead for network and it is not suitable for upper layer. In [6] wireless mesh network high throughput need a TDMA based approach. TDMA support multichannel transmission, schedule dissemination and routing integration. TDMA based on routing metrics and stability of routing metrics. Experiment shows that it control the network overhead and it not affected by external interference. It is not useful for large network. In [3] TDMA each frame is associated with some slot, and non -conflicting link is transmitted through these slots. Iterative procedure is used to find the nearest feasible schedule by exchanging the link information between nodes. Another part is work on wave based termination which is used to detect the scheduled nearest node and if any new node is scheduled which is activated. SpatialTDMA [9] used for reduced the energy consumption and improve the throughput author formulate offline energy -throughput by the tradeoff curve. Physical interference involved where node used for controlling the power. Author work is based on single channel or single node, it is not feasible for multi-channel or multiple node scenario. Here [7] author works for high throughput and reliable mesh network in multi-hop transmission. Reduce network bandwidth in

multicast tree. Author presents a distributed and centralized algorithm for tackle the problem of multicasting. Obtained result from expected multicast transmission count (EMTX) method, 
shows the effectiveness it reduces the number of hop-by-hop transmission per packet, but it not considerable for real life or realistic scenario. A hierarchical mesh tree protocol given in [10] which achieve efficient routing at the MAC layer. Optimal route is chosen by the using of mesh tree topology. The used new HMTP also used for maintaining the update of new route formed. The HMTP topology overcomes the drawback cluster-tree. In[8] improvement of QoS by avoiding the network congestion, an algorithm is designed for that prediction of congestion is done before it really happen by using different data, analysis of network and used the historical data of traffic to generate idea of future network traffic. Through network traffic data, load balancing is done to avoid congestion in wireless mesh network. But author proposed algorithm is inefficient to response network congestion properly.

\subsection{Issues and Challenges Faced in Multi Radio Wireless Mesh Network}

Using IEEE 802.15.5 standard WMN in free frequency bands for wireless communications traffic has the following issue the need to be addressed as in [12]:

Speed of mobile devices. Physical and transport levels of IEEE 802.15.5 developed for fixed stations, where high speed can lead to large and rapid changes in channel conditions, which in turn increases the probability of frame error (FER). This occurrence is due to the Rayleigh fading channel [11].

Distance: IEEE 802.15.5 is used for communications over short distances of several hundred meters. Together with this limitation there is a necessity in a large number of access points/roadside stations (remote control) to cover the entire route. Usually 802.15.5 transmits a plurality of control frames and messages for the association and/or network authentication before transmitting useful information.

Handover is also difficult to implement due to the high speed of vehicles on the road, where the handoff occurs very frequently between the remote control for the entire route.

When designing a Mesh network appear the following difficulties:

Difficult to predict the number of subscribers on the network at different intervals. Difficulties in predicting the amount of traffic generated by nodes, hence the total system capacity. The wireless channel is stochastic and time-varying according to different parameters.

It is seen from literature that the existing mac based mesh network suffer improper channel slot re-utilization. To overcome the short coming here the author propose an efficient cross layer based channel slot re-utilization optimization based on node classification technique to improve the QoS of WMNs.

The paper organization is as follows: The proposed channel slot re-utilization models are presented in Section two. The results and the experimental study are presented in the section three. The concluding remark is discussed in the last section.

\section{PROPOSED MODEL}

Here the author proposes an adaptive cross layer based slot channel re-utilization optimization to improve the QoS of WMNs. The slot re-utilization helps in reducing latency of data delivery LP 
LP but there is a chance or risk of beacon collision to some other devices that may join late to the wireless mesh network. To address this here the author proposes a node classification based slot re-utilization technique to reduce latency and propose robust wireless mess tree architecture.

\subsection{Proposed Node Classification for Slot Re-utilization for WMN}

Here the author classifies the device pair based on the information of parenthood and neighbourhood relationship. The classified device pair $(\mathrm{x}, \mathrm{y})$ is as follows

\subsubsection{Connected Inner Relay Node Pair (CIRP):}

Here $\mathrm{x}$ and $\mathrm{y}$ are adjacent device that exist physically in the WMN, and either $\mathrm{x}$ and $\mathrm{y}$ are not adjacent device; or, either $\mathrm{x}$ or $\mathrm{y}$ has a child, but xand $\mathrm{y}$ have a conjoint adjacent device which is a child of either $\mathrm{x}$ or $\mathrm{y}$.

\subsubsection{Connected Leaf Relay Node Pair (CLRP):}

Here $\mathrm{x}$ and $\mathrm{y}$ are adjacent devices that exist physically in the WMN, but neither $\mathrm{x}$ nor $\mathrm{y}$ has any child.

\subsubsection{Distant Inner Relay Node Pair (DIRP):}

Here $\mathrm{x}$ and $\mathrm{y}$ are not adjacent devices physically but have a conjoint adjacent physical devices in common, although all these adjacent physical devices are neither x's nor y's children.

\subsubsection{Non-connected leaf node pair (NCLRP):}

Here $\mathrm{x}$ and $\mathrm{y}$ are not adjacent physical devices, neither do they have adjacent physical devices in common. Now the author propose channel slot reutilization by CLRP, DIRP and NCLRP when the probability of is risk is small which is explained in below section.

\subsection{Proposed Slot Re-Utilization for WMN}

The important part of our technique is to compute the risk probability of slot re-utilization for a specified pair of devices $(x, y)$ based on the current parenthood and neighbourhood knowledge. The author does not consider the knowledge of the physical distance among $x$ and $y$ or the total number of devices. The author consider that the WMN node is deployed in a random manner yet follows even or uniform distribution over a section $S$. Let $R$ be the area of $S$. Each node is presumed to have a range of communications range of ${ }^{S}$. A section of area in $S$ is assumed to be enclosed by a node ${ }^{x}$ and is represented as ${ }^{\prime} s$ coverage if all the point in this network area is within range of communications of $x$. The author assumes that $V(x, y)$ to signify the area of the section concealed by two nodes $x$ and $y$, and use $l(x, y)$ to signify the physical distance among them.

It is known fact that the radio coverage of a wireless transmitter suffer from path loss phenomenon. A practical radio propagation model may consider random variations in path loss at 
different direction and location [13] and [14], [16] respectively. Subsequently the packet transmission among pair of devices becomes a probabilistic task with probability distribution utility considering direction or distance among the transmitter and the receiver as a parameter. The proposed node or device-pair classification and communication evaluation are based on the perception of adjacent physical devices, which represent a binary association considering that the two nodes are either adjacent physical devices or are not. Such an association needs a setup of a thresholding on packet or data reception probability for the decision of adjacent physical devices in accordance to a source or transmitter. Subsequently the sources or transmitter operative range of communications would be uneven. However, we can estimate the lower bound of the range of transmission within which any nodes can have an association with the source or transmitter, or a higher of the range, outside which no nodes will be able to receive the data from sources or transmitter successfully.

The symbolization $s$ is represented as the lower bound, the upper bound, or the average bound of range of communication, in which case it is essentially the worst case, best case or the mean case risk parameters of the analytical result presented in the following sturdy.

\subsubsection{Scheme 1:}

The predictable mesh area of the particular region mutually covered by two devices $x$ and $y$ is

$$
\begin{aligned}
& G|V(x, y)|=\left\{\left(\pi-\frac{3 \sqrt{3}}{4}\right) \text { if } p(x, y) \leq s .\right. \\
& G|V(x, y)|=\left\{\frac{\sqrt{3}}{4} s^{2} \text { if } s<p(x, y) \leq 2 s .\right.
\end{aligned}
$$

A device that is positioned at the edge of $S$ is expected to cover less area than as part of its coverage in beyond or outside $S$. This effect is known as the border effect. To overcome the impact by the border effect the author presents the following analysis considering that the region covered by any device is within $S$. If $S$ represents a rectangle area, the probability can be evaluated by adopting the torus convention methodology [15], which turns a flat rectangle into a torus. With this theory, the link occurrence probability [17] is formulated as $p=\pi s^{2} / R$. Our fundamental outcome consists of following three Propositions which is presented below.

\subsubsection{Proposition 1:}

Consider that the device are distributed uniformly over a region $S$ of $R$ size, and $R \gg \pi s^{2}$. Assume a device pair $(x, y) \in Y P$ are using same channel slot when a new device ${ }^{n}$ join a network which is represented by $P_{Y}(x, y)$. The predictable probability of ${ }^{n}$ suffer from the risk involved in channel slot re-utilization among device $x$ and $y$ is $P_{Y}(x, y)=\left(1-\frac{3 \sqrt{3}}{4 \pi p}\right)$ where $p=\pi s^{2} / R$. 
Proposition 1 is verified based on the inclusion exclusion principle and the outcome of Scheme 1 . It shows that $P_{Y}=(x, y) \approx 1.41 p$, therefore increase in range of communication involves high probability of collision among devices in $Y P$.

\subsubsection{Proposition 2:}

Proposition 2 represented as $P_{Z}=(x, y)\left(\operatorname{resp} . P_{X}(x, y)\right)$ is the probability expected when a device $n$ joining a network becomes a victim of slot re-utilization among $x_{\text {and }} y$ when $(x, y) \in Z P($ resp. $(x, y) \in X P)$. Moreover the predictable probability $P_{X}^{*}(x, y)$ that a device ${ }^{n}$ joining a network become a fatality or victim of channel slot re-utilization among ${ }^{x}$ and $y_{\text {when }}(x, y) \in X P_{\text {considering that }} s<d(x, y) \leq 2 s$. Let $a_{\text {represent the number of adjacent }}$ device of $y$, then $P_{Z}(x, y)$ and $P_{X}(x, y)$ are correlated to $P_{X}^{*}(x, y)$ by

$P_{z}(x, y)=\frac{\sqrt{3}}{4 \pi(1-\varphi(a))} p-\frac{\varphi(a)}{1-\varphi(a)} P_{X}^{*}(x, y)$,

$$
P_{X}(x, y)=3 p P_{X}^{*}(x, y),
$$

Where $p=\pi s^{2} / R$ and

$$
\varphi(a)=\frac{2}{3} \int_{\theta=0}^{2 \pi / 3}\left[1-\frac{\theta-\sin \theta}{\pi}\right]^{a} \sin \theta d \theta .
$$

Function $\varphi(a)$ is the predictable probability of all $y^{\prime} s$ adjacent devices not residing in the region covered by ${ }^{x}$ and $y$ considering that $s<d(x, y) \leq 2 s$. Subsequently $\varphi(a)$ is reducing in accordance to value of $a, \varphi(a) \leq \varphi(1)=1-\frac{\sqrt{3}}{4 \pi}$ and $1-\varphi(a) \geq 1-\varphi(1)=\frac{\sqrt{3}}{4 \pi}$ for all $a \geq 1$. It follows the first term of (1) is lesser than $1 p$ and $P_{Z}(x, y)$ hence it is upper bounded by $p_{\text {for the case of }} a=1$.

It follows that

$$
P_{Z}(x, y)=p-\frac{1-\frac{\sqrt{3}}{4 \pi}}{\frac{\sqrt{3}}{4 \pi}} P_{X}^{*}(x, y) \geq 0 .
$$

$$
P_{X}^{*}(x, y) \leq \frac{\sqrt{3}}{4 \pi-\sqrt{3}} p \approx 0.16 p
$$

Therefore $P_{X}(x, y)=3 p P_{X}^{*}(x, y)=0.48 p^{2}$ 
For a general $a$, the integration task of $\varphi(a)$ requires an intensive computation procedure. Luckily the value of $\varphi(a)$ corresponding to practical value os $a$ are pre-calculated and kept in the devices before deployment. These assessment can be checked when $\varphi(a)$ need to be computed. More notably based on Proposition 2 we can evaluate $P_{X}^{*}(x, y)$, then we can predict the precise value of $P_{Z}=(x, y)$ and $P_{X}(x, y)$. Next based on Proposition 3 we evaluate the $P_{X}^{*}(x, y)$.

\subsubsection{Proposition 3:}

Based on the definition of Proposition 2,

$$
P_{X}^{*}(x, y)=\int_{s}^{2 s} V(b)\left\{\frac{\left[1-\frac{V(x)}{\pi s^{2}}\right]^{a} \frac{2 s}{3 s^{2}}}{\int_{s}^{2 s}\left[1-\frac{V(x)}{\pi s^{2}}\right]^{a} \frac{2 s}{3 s^{2}}}\right\} d x
$$

Where

$$
V(b)=s^{2}\left[2 \arccos \frac{b}{2 s}-2 \sqrt{1-\left(\frac{b}{2 s}\right)^{2}}\left(\frac{b}{2 s}\right)\right]
$$

From Figure 1 and Figure 2 Compares the values of $P_{Z}(x, y)$ and $P_{X}(x, y)$ respectively are the simulation value obtained based on Proposition 2 and Proposition 3. The simulation effective range of communication is set and the areas are fixed as $\sqrt{a R / c \pi}$, and devices are added to the simulation bed until an average degree ${ }^{a}$ of a device is reached. Our analytical or mathematical result gives a high precision of accuracy. Proposition 3 requires an intensive computation for increase in $a$. For a device to compute $P_{X}^{*}(x, y)$ effectively here the author propose the following approximation

$P_{Z}(x, y) \approx 0.17 p$.

The value of $P_{X}(x, y)$ are largely so low and are considered to be zero i.e.... $P_{Y}(x, y) \geq P_{Z}(x, y) \geq P_{X}(x, y)$ or $P_{X}(x, y) \geq P_{Z}(x, y) \geq P_{X} \geq P_{Y}(x, y)$.

The simulation sturdy of our proposed approach is evaluated in the below section of this paper.

\section{Simulation RESULT AND ANALYSIS}

The system environment used is windows 10 enterprises 64-bit operating system with 16GB of RAM. The author have used visual studio Dot net framework 4.0, 2010 and used VC++ programming language. The author has conducted simulation study for probability analysis for channel slot re-utilization by varying the node size and node degree. 
In Fig. 1, the author computes the probability of $\mathrm{P} \_\mathrm{Z}(\mathrm{x}, \mathrm{y})$ by varying number of node or devices and node degree value. In figure we can see that when we increase the node degree a value the probability of collision also increases for all node sizes $(50,100$, and 150). The probability of collision is high for smaller mesh network size (50) when compared to larger network size (150).

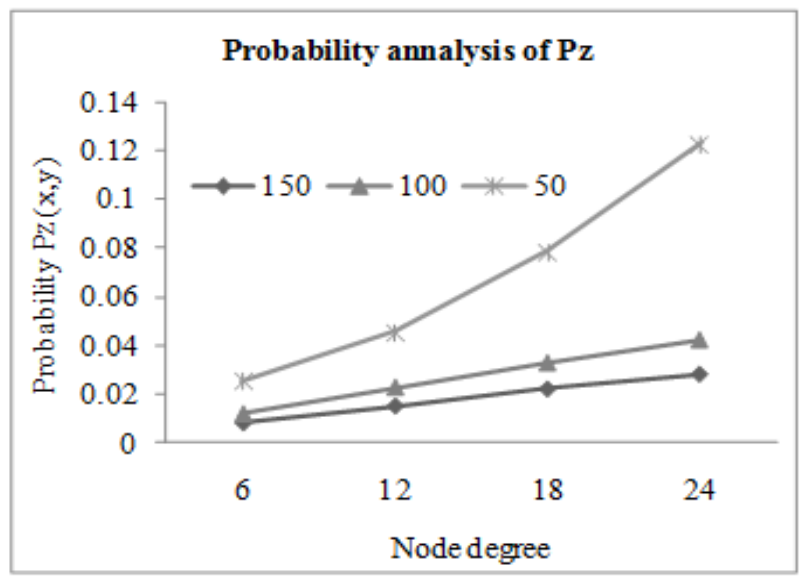

Figure 1. Probability analysis for $P_{Z}(x, y)$

In Fig. 2, the author computes the probability of $\mathrm{P}_{-} \mathrm{X}(\mathrm{x}, \mathrm{y})$ by varying number of node or devices and node degree value. In figure we can see that when we increase the node degree a value the probability of collision also increases for all node sizes $(50,100$, and 150). The probability of collision is high for smaller mesh network size (50) when compared to larger network size (150).

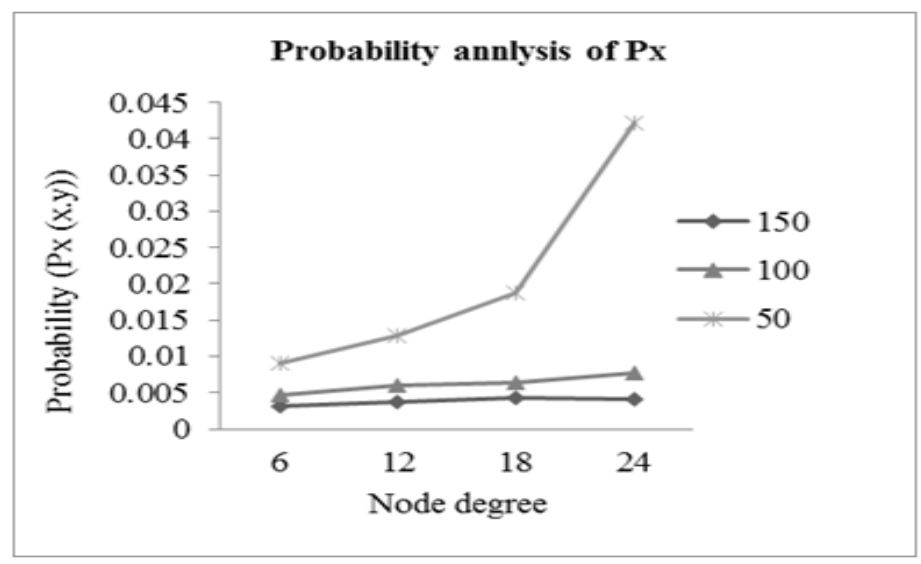

Figure 2. Probability analysis for $P_{X}(x, y)$ 


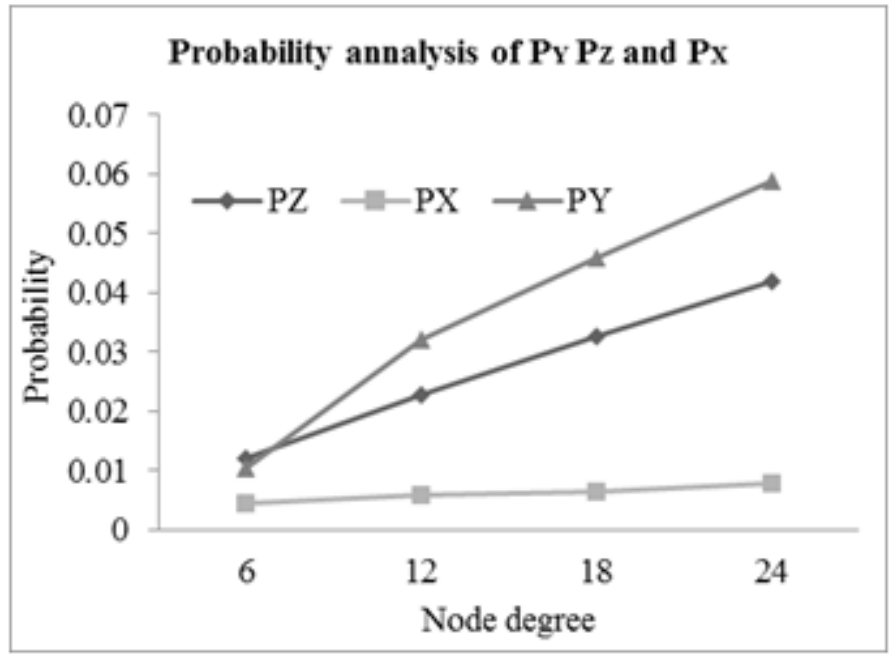

Figure 3. Probability analysis

In Fig. 3, the author computes the probability of $P_{X}(x, y) P_{Z}(x, y) P_{Y}(x, y)$ by node degree value for 150 nodes or devices. In figure we can see that when we increase the node degree $a$ value the probability of collision also increases. The probability of collision is high for $P_{Y}(x, y)$, the probability of collision is low for $P_{X}(x, y)$ and the probability of collision for $P_{Z}(x, y)$ lies between $P_{X}$ and $P_{Y}$. Thus proving proposition $3 P_{Y}(x, y) \geq P_{Z}(x, y) \geq P_{X}(x, y)$.

\section{CONCLUSIONS}

The paper presents a model that help in the design of MWNs that meets the QoS necessities of the end user. Here in this work we have presented a cross-layer based channel slot reutilization model based on node classification technique that minimizes the collision for channel slot reutilization and thus helps in reducing latency for data transmission in WMN. The experimental result shows the impact of proposed model on channel slot re-utilization. In future we would conduct simulation sturdy for latency and compare our proposed model with other distributed, centralized or MAC based scheduling algorithm.

\section{REFERENCES}

[1] Chin-Ya Huang; Ramanathan, P., "Network Layer Support for Gigabit TCP Flows in Wireless Mesh Networks," in Mobile Computing, IEEE Transactions on , vol.14, no.10, pp.2073-2085, 2015.

[2] Anusha, M.; Vemuru, S.; Gunasekhar, T., "TDMA-based MAC protocols for scheduling channel allocation in multi-channel wireless mesh networks using cognitive radio," in Circuit, Power and Computing Technologies (ICCPCT), International Conference on , vol., no., pp.1-5, 2015.

[3] Djukic, P.; Valaee, S., "Distributed Link Scheduling for TDMA Mesh Networks," in Communications, 2007. ICC '07. IEEE International Conference on, vol., no., pp.3823-3828, 24-28 2007. 
[4] Yu Cheng; Hongkun Li; Peng-Jun Wan; Xinbing Wang, "Wireless Mesh Network Capacity Achievable Over the CSMA/CA MAC," in Vehicular Technology, IEEE Transactions on , vol.61, no.7, pp.3151-3165, 2012.

[5] Teymoori, Peyman; Yazdani, Nasser; Khonsari, Ahmad, "DT-MAC: An Efficient and Scalable Medium Access Control Protocol for Wireless Networks," in Wireless Communications, IEEE Transactions on , vol.12, no.3, pp.1268-1278, 2013.

[6] Sevani, V.; Raman, B.; Joshi, P., "Implementation-Based Evaluation of a Full-Fledged Multihop TDMA-MAC for WiFi Mesh Networks," in Mobile Computing, IEEE Transactions on , vol.13, no.2, pp.392-406, 2014.

[7] Xin Zhao; Jun Guo; Chun Tung Chou; Misra, A.; Jha, S.K., "High-Throughput Reliable Multicast in Multi-Hop Wireless Mesh Networks," in Mobile Computing, IEEE Transactions on , vol.14, no.4, pp.728-741, April 12015.

[8] Khasawneh, F.A.; Benmimoune, A.; Kadoch, M.; Khasawneh, M.A., "Predictive Congestion Avoidance in Wireless Mesh Network," in Future Internet of Things and Cloud (FiCloud), 2015 3rd International Conference on , vol., no., pp.108-112, 24-26 Aug. 2015

[9] Ouni, A.; Rivano, H.; Valois, F.; Rosenberg, C., "Energy and Throughput Optimization of Wireless Mesh Networks With Continuous Power Control," in Wireless Communications, IEEE Transactions on , vol.14, no.2, pp.1131-1142, 2015.

[10] Rabarijaona, V.H.; Kojima, F.; Harada, H., "Hierarchical mesh tree protocol for efficient multi-hop data collection," in Wireless Communications and Networking Conference (WCNC), 2014 IEEE , vol., no., pp.2008-2013, 6-9 April 2014.

[11] Safronov, R.; Bakhtin, A.; Muravyev, I.; Muratchaev, S., "Designing roadside mesh network with TDMA," in Ultra-Modern Telecommunications and Control Systems and Workshops (ICUMT), vol., no., pp.131-135, 2014.

[12] K. Bilstrup. A survey regarding wireless communication standarts intended for a high-speed mobile environment// Technical Report IDE, 2007.

[13] G. Zhou, T. He, S. Krishnamurthy, and J.A. Stankovic, "Models and Solutions for Radio Irregularity in Wireless Sensor Networks," ACM Trans. Sensor Networks, vol. 2, no. 2, pp. 221-262, 2006.

[14] F. Kuhn, R. Wattenhofer, and A. Zollinger, "Ad Hoc Networks beyond Unit Disk Graphs," Wireless Networks, pp. 715-729, 2008.

[15] P. Hall, Introduction to the Theory of Coverage Processes. John Wiley and Sons, 1988.

[16] I. Stojmenovic, A. Nayak, and J. Kuruvila, "Design Guidelines for Routing Protocols in Ad Hoc and Sensor Networks with a Realistic Physical Layer," IEEE Trans. Comm., vol. 43, no. 3, pp. 101-106, 2005.

[17] L.-H. Yen and Y.-M. Cheng, "Clustering Coefficient of Wireless Ad Hoc Networks and the Quantity of Hidden Terminals," IEEE Comm. Letter, vol. 9, no. 3, pp. 234-236, 2005. 


\section{AUTHORS}

Asha C N received the Bachelor of Engineering degree in Electronics and Communication Engineering from Bangalore University in 2002 and Master of Technology degree in VLSI Design \& Embedded from Visvesvaraya Technological University (VTU) in 2008.She is an assistant professor in Electronics and Communication Engineering Department, Acharya Institute of Technology since 2007. Her research interest includes Wireless Mesh Network (WMN), Cross Layer Design and Routing protocols. She is life member of Indian Society of Technical Education(ISTE).

T G Basavaraju received Ph.D. (Engg) from Jadavpur University, Kolkata in the area of Mobile Adhoc Networks. He obtained his Master Degree in Computer Science and Engineering from Visveswaraya College of Engineering (UVCE), Bangalore University. He holds Bachelor's degree in Computer Science and Engineering from Kuvempu University. His areas of research are Wireless Adhoc Networks, Sensor Networks and Mesh networks. He has to his credit more than 55 research publications in National/International Journals and Conferences. He is Professor and Head of Computer Science and Engineering Department at Government SKSTI, Bangalore and

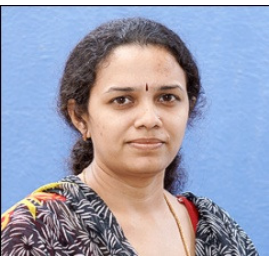
has 20 years of experience

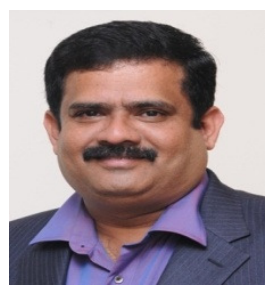
. 